\title{
Gluteus as a rare localization of extragonadal teratoma
}

\author{
Davide Leardini ${ }^{1}$, Sara Cerasi ${ }^{2}$, Maria Elena Cantarini ${ }^{2}$, Elena Facchini ${ }^{2}$, Arcangelo Prete ${ }^{2}$, \\ and Riccardo Masetti ${ }^{3}$ \\ ${ }^{1}$ University Hospital of Bologna Sant'Orsola-Malpighi Polyclinic \\ ${ }^{2}$ IRCCS Azienda Ospedaliero-Universitaria di Bologna Policlinico S Orsola-Malpighi \\ ${ }^{3}$ University of Bologna Hospital of Bologna Sant'Orsola-Malpighi Polyclinic
}

February 16, 2022

\section{TITLE PAGE}

\section{GLUTEUS AS A RARE LOCALIZATION OF EXTRAGONADAL TERATOMA}

Authorship: Leardini Davide ${ }^{1}$, Cerasi Sara ${ }^{1}$, Cantarini Maria Elena ${ }^{1}$, Facchini Elena ${ }^{1}$, Prete Arcangelo ${ }^{1}$, Masetti Riccardo ${ }^{1}$

1 Pediatric Oncology and Hematology Unit "Lalla Seràgnoli", Istituto di Ricovero e Cura a Carattere Scientifico (IRCCS), Azienda Ospedaliero-Universitaria di Bologna, Bologna, Italy

Correspondence: Sara Cerasi; address: via G. Massarenti 11, 40138, Bologna (BO), Italy; phone: +39 051 2144665; e-mail:sara.cerasi@studio.unibo.it

Word Count for Main Text: 547 words

Attached to this manuscript there is 1 figure

Keywords: extragonadal teratoma, gluteal mass, immature teratoma

Running title: Rare non-midline teratoma

To the Editor,

Teratomas represent the most common germ cell tumors in children ${ }^{1}$. They can be gonadal, more common in adolescents, or extragonadal, primarily in neonates and young children. Teratomas develop from totipotent primordial cells and may originate anywhere along the midline. Common sites for extragonadal teratomas are the sacrococcygeal region, which accounts for $35-60 \%$ of all teratomas, the mediastinum, the retroperitoneum, the head and neck and the central nervous $\mathrm{system}^{2-4}$. Other localizations are rare, especially non-midline ones that are very often lateralized expansions of midline teratomas, such as those arising from sacrococcygeal region.

We here describe the case of a newborn girl presenting with a gluteal mass, that revealed to be a primary extragonadal teratoma. At birth she presented with a hard-elastic, mobile and painless mass localized within the right gluteus (Fig.1), that had not been noted on prenatal ultrasound. At two days of life, an echography was performed, revealing a subcutaneous irregularly hypoechoic mass with fluid areas inside and small vessels, with aspecific characteristics. The dimension of the mass was $24 \times 15 \mathrm{~mm}$ and rapidly increased in size, reaching $40 \times 25 \mathrm{~mm}$, and in the number of fluid areas (Fig. 1). Alfafetoprotein serum concentration resulted $4586 \mathrm{ng} / \mathrm{mL}$ (refence value at the 2 week- 1 month interval at which she was tested $316-6310 \mathrm{ng} / \mathrm{mL}$ ) and $\mathrm{hCG}$ was $0,8 \mathrm{UI} / \mathrm{L}$ (normal value $<5 \mathrm{UI} / \mathrm{L})^{5}$. After performing an MRI that excluded other lesions, the mass was removed and a biopsy was performed, revealing an immature teratoma, grade 3 according to 
Norris's classification. Since extragonadal teratomas out of the midline are very rare, she has been followed up thoroughly for 3 years with regular periodic blood tests and radiological assessments, but no other primary lesions or recurrencies were found.

Teratomas can be malignant (12-14\%) or benign, further divided into mature (50-60\%) and immature (18$34 \%$ ). Immature teratomas contain fetal tissue, most often neuroectodermal, the amount of which is scored according to a grading system introduced by Norris. Grade 3 is that with most neuroectodermal tissue, and have an increased incidence of local recurrence and malignant degeneration ${ }^{3,4,6}$. Complete and prompt surgical resection is the gold standard for definitive therapy in benign teratomas, both mature and immature ${ }^{4,6}$.

Teratomas develop along the midline because they originate from the incomplete differentiation of totipotent primordial cells that arise in the yolk sac and migrate along the mesentery to the gonadal ridge during the $4^{\text {th }}-5^{\text {th }}$ week of embryologic development ${ }^{3,4}$. Indeed, most of the gluteal teratomas reported in literature are lateralized sacrococcygeal teratomas with a connection to the coccyx, since sacrococcygeal teratomas are thought to be derived from totipotent cells of the Hensen's node (primitive knot), an area at the cranial end of the primitive streak ${ }^{7,6}$.

Other authors reported rare sites for lateralized teratoma development such as kidney, liver and temporozygomatic region ${ }^{8,9}$. Rare lateralized extragonadal localizations should not mislead the clinical suspicion of teratomas, and a primary localization should always be excluded. Taking also the patient's age into account, it can sometimes be considered to perform a PET scan. To the best of our knowledge, there is just one case in literature of a gluteal teratoma not in connection with the coccyx, as in our patient, thus confirming the possibility of this very rare localization ${ }^{10}$. The biological mechanism for germ-cell migration in such anatomical regions is still to be elucidated.

ACKNOWLEDGEMENTS: None.

CONFLICT OF INTEREST

The authors declare that there is no conflict of interest.

\section{ETHICS STATEMENT}

Written informed consent has been obtained from the patient to publish this paper.

\section{References}

1. Shaikh F, Murray MJ, Amatruda JF, et al. Paediatric extracranial germ-cell tumours. Lancet Oncol . 2016;17(4):e149-e162. doi:10.1016/S1470-2045(15)00545-8

2. Terenziani M, D'Angelo P, Inserra A, et al. Mature and Immature Teratoma: A Report From the Second Italian Pediatric Study.Pediatr Blood Cancer . 2014;62:1202-1208. doi:10.1002/pbc.25423

3. Lakhoo K. Neonatal teratomas. Early Hum Dev . 2010;86(10):643-647. doi:10.1016/j.earlhumdev.2010.08.016

4. Barksdale EM, Obokhare I. Teratomas in infants and children. Curr Opin Pediatr . 2009;21(3):344-349. doi:10.1097/MOP.0b013e32832b41ee

5. Blohm MEC, Vesterling-Horner D, Calaminus C, Gobel U. Alpha-fetoprotein (AFP) reference values in infants up to 2 years of age. Pediatr Hematol Oncol . Published online 1998:135-142.

6. Yoon HM, Byeon SJ, Hwang JY, et al. Sacrococcygeal teratomas in newborns: a comprehensive review for the radiologists. Acta radiol . 2018;59(2):236-246. doi:10.1177/0284185117710680

7. Aihole JS. A Vague Gluteal Swelling in a Neonate: Challenges in Its Diagnosis and Management. Short Title-Sacroccoygeal Teratoma. Glob Pediatr Heal . 2020;7:0-3. doi:10.1177/2333794X20982438 
8. Paradies G, Zullino F, Orofino A, Leggio S. Rare extragonadal teratomas in children: complete tumor excision as a reliable and essential procedure for significant survival. Clinical experience and review of the literature. Ann Ital Chir . 85(1):56-68.

9. Bhalla S, Masih K, Rana RS. Teratomas of rare sites: a review of ten cases. J Indian Med Assoc . 1991;89(10):291-294.

10. Dutta HK, Borah P, Baruah M. Gluteal teratoma: A rare site of extragonadal teratoma. J Indian Assoc Pediatr Surg . 2016;21(4):178-180. doi:10.4103/0971-9261.158097

\section{LEGENDS}

Figure 1: A Picture of the gluteal mass, B MRI image of the immature teratoma

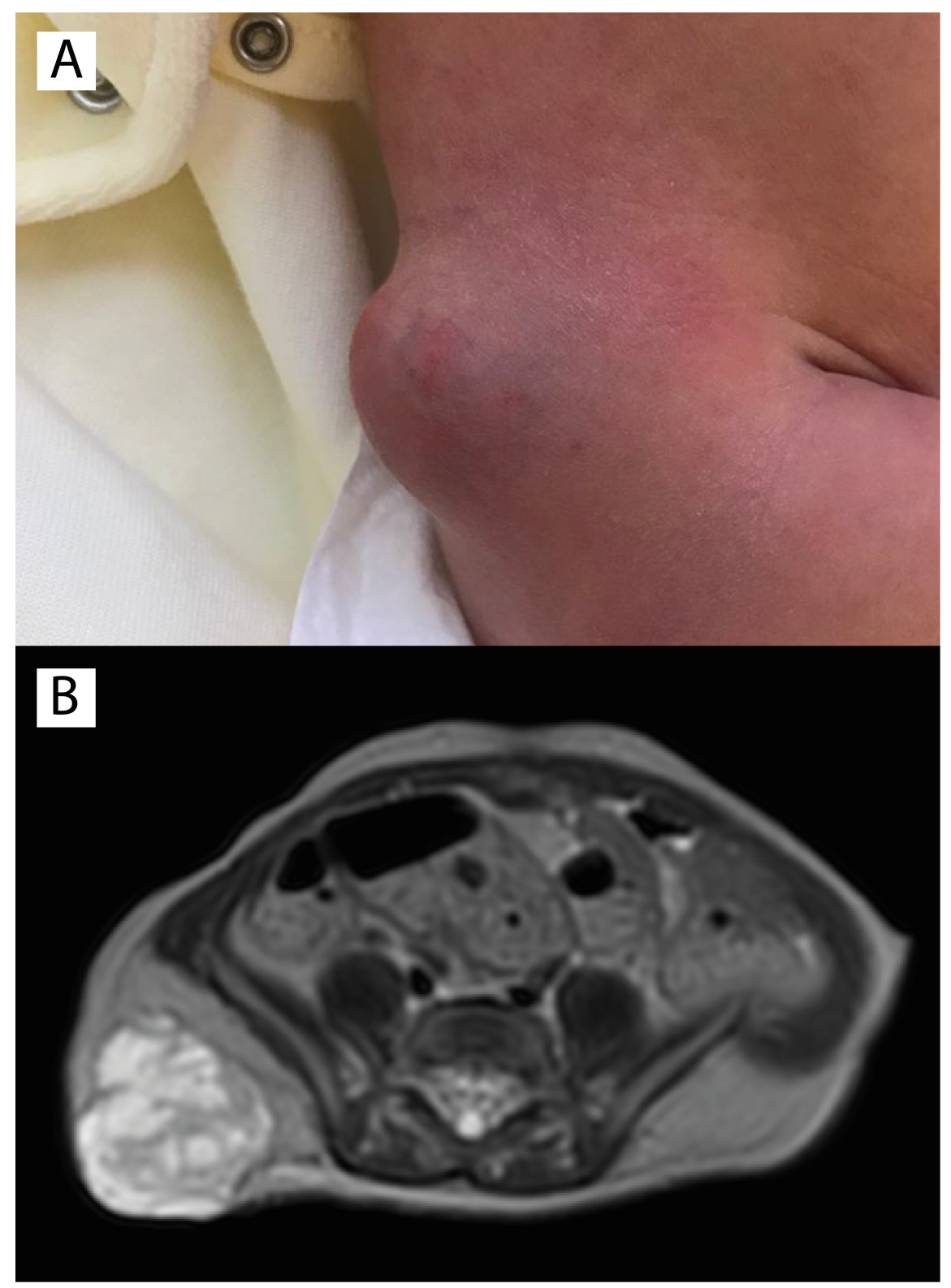

